\title{
Innovación y Emprendimiento Empresarial: dos caras de una misma moneda
}

\section{Innovation and Entrepreneurship: two faces of the same currency}

\author{
MSc. Irina Naranjo Bert ${ }^{1}$ \\ inaranjobert@gmail.com
}

PhD. Daniel Claudio Perazzo Logioia ${ }^{2}$

danielclaudio.perrazzo@gmail.com

Recibido: 1/12/2019; Aceptado: 4/3/2020

\begin{abstract}
RESUMEN
Los procesos de emprendimiento empresarial e innovación son dos caras de la misma moneda. La innovación permite que una idea creativa se ponga en práctica y resuelva problemas concretos ante una carencia o demanda, el emprendimiento lo llevan a cabo personas que tienen como premisa cumplir sus sueños, muchas veces motivados por entornos altamente dinámicos, con pocas ofertas de empleo y bajos salarios. Esta idea, que surgió como la solución ante ese entorno hostil, o ante ese deseo de independencia, puede llegar a concretarse igualmente en una oferta de gran valor para sus clientes y en mayores beneficios para sus trabajadores y la comunidad. Ambos procesos implican creatividad, empatía, valentía y arrojo. Uno puede llegar a ser consecuencia del otro; la innovación genera emprendimientos y éstos a su vez, siguen generando innovaciones, ambos pueden conducir, con la guía y responsabilidad adecuadas, a una mejor calidad de vida de la población y la sociedad en general.
\end{abstract}

Palabras clave: Idea, creatividad, innovación, emprendimiento, valor

\footnotetext{
1 Instituto Superior Tecnológico Particular Sudamericano, Cuenca. Ecuador

2 Instituto Superior Tecnológico Particular Sudamericano, Cuenca. Ecuador
} 


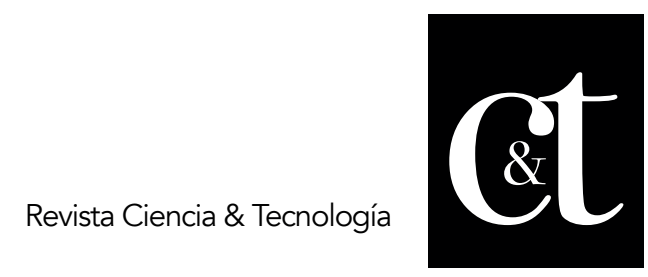

No. 26, 30 de abril de 2020

ISSN impreso: 1390 - 6321

ISSN online: 2661 - 6734

\begin{abstract}
The processes of business entrepreneurship and innovation are two sides of the same coin. Innovation allows a creative idea to be put into practice and solve specific problems in the face of a lack or demand, entrepreneurship is carried out by people whose premise is to fulfill their dreams, often motivated by highly dynamic environments, with few job offers and low wages. This idea, which emerged as the solution to this hostile environment, or to that desire for independence, can also come to fruition in an offer of great value to its customers and in greater benefits for its workers and the community. Both processes involve creativity, empathy, courage, and courage. One can become a consequence of the other; innovation generates entrepreneurship and these in turn, continue to generate innovations, both can lead, with the appropriate guidance and responsibility, to a better quality of life for the population and society in general.
\end{abstract}

Keywords: idea, creativity, innovation, entrepreneurship, value

\title{
Introducción
}

El entorno empresarial actual se presenta cambiante, dinámico y muy competitivo, ya no es suficiente conocer que demanda el mercado, sino que hay que ser capaz de ser visionario y tener una actitud proactiva ante el mismo, de esta manera, la mayoría de los países, con especial énfasis en los que están en vías de desarrollo, necesitan de empresarios emprendedores y de procesos innovadores en todos los ámbitos de la economía y la sociedad. Muchos gobiernos, al darse cuenta que la oferta de mano de obra excede la demanda de trabajo, preparan programas de apoyo para que, un porcentaje importante de la población, inicie su propio negocio y de esta manera propiciar la reducción de los niveles de desempleo.

Con el aumento de los emprendimientos, la mayoría de ellos empresariales, se incrementa la competencia en determinados sectores, sobre todo en aquellos en los que las barreras de entrada son menores, por lo que constituye un verdadero reto lograr vender sus ofertas y hacer crecer sus emprendimientos. En este sentido, cobra vital importancia saber detectar a tiempo qué oferta de valor satisfará mejor al mercado. De esta forma, la mentalidad del emprendedor y su equipo, debe ser creativa, capaz de generar soluciones rápidas, siendo la innovación, el proceso que va a ayudar a identificar y a entregar al mercado aquella oferta diferente, útil y oportuna, para garantizar la permanencia de estas organizaciones a lo largo del tiempo.

\section{Implicaciones de la innovación}

La innovación es un proceso que implica la utilización del conocimiento para crear nuevas soluciones ante carencias o demandas que se quieran satisfacer, y que conlleven, por tanto, a un aumento de valor y a una mayor prosperidad en la sociedad. Hay que atender, como paso importante, a los modelos e innovación (Pizzi y Brunet, 2013). Según Domínguez (2004) solo se puede hablar de innovación si esta mejora, cambio o desarrollo, concluye con su éxito en el mercado, por lo que, si nadie está dispuesto a pagar por esta oferta, si no se perciben estos beneficios por el mercado, no hay una innovación. Perazzo, D. (1996) afirma que "La Innovación implica la oportunidad para realizar cambios estratégicos en la organización y administración de una empresa, a partir de la 


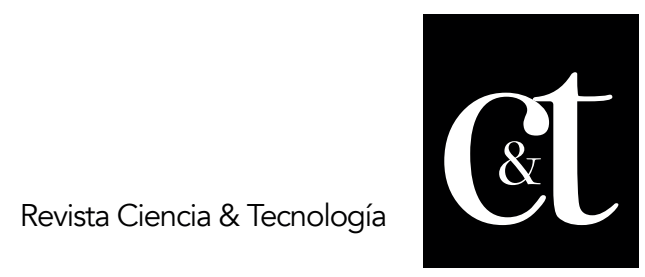

No. 26, 30 de abril de 2020

ISSN impreso: 1390 - 6321

ISSN online: 2661 - 6734

experiencia y tomando como criterios la transformación y los requerimientos del entorno con el propósito de incrementar la cultura organizacional". Con ello, además estamos apostando a una de las principales tendencias de la administración moderna, impulsando la evolución de la misma y fortaleciendo el "Downsizing", con lo cual se da un giro de $360^{\circ}$ en la empresa, puesto que permite incrementar la productividad y convertirla en más eficiente.

En la compleja dinámica del entorno económico actual, innovar no es sólo crear lo nuevo, sino además enriquecer lo existente. De esta forma, se habla de innovación cuando se renueva la gama de bienes y servicios actuales, cuando cambian los procesos productivos e incluso cuando cambia el modo en que se gestiona una organización. Detrás de la creación de nuevas soluciones que posibiliten mayores beneficios en el plano económico, social y tecnológico, está la innovación. Para que la innovación sea efectiva, las ideas creativas deben surgir condicionadas por su contexto, y luego deben ser implementadas con el éxito suficiente para que logren resolver la problemática planteada de forma que aporten un progreso que antes no existía sin esta solución.

Las preguntas caen por su propio peso en el sentido del emprendimiento en mayor o menor grado por parte de los empresarios (Fuentelsaz y Montero (2015). Además, todo puede ser mejorado, solo habría que hacerse las preguntas correctas: "¿qué puedo combinar, adaptar, eliminar, agrandar o reducir, sustituir, reacomodar o invertir, alterar, utilizar en otra forma y anticipar en el futuro para mejorar lo que me proponga?" (Schoening, 2017, p. 58). Este autor explica cada una de estas interrogantes de la siguiente manera; combinar es mezclar dos cosas, ejemplos claros se ven en el área gastronómica, cuando se combinan alimentos y se forman nuevos platos, adaptar implica ajustar; los tapones que se ajustan a la entrada de una botella, las cejas de las latas de conservas o las cejas de los envases de refrescos que se transformaron para abrirlos rápidamente. Al eliminar se quita definitivamente, separa o descarta, también forma parte de esos cambios o mejoras si implican al final un mejor bien o servicio, ejemplo cuando se eliminan gastos innecesarios, procesos o actividades que lejos de incrementar el valor de la oferta lo disminuye. Y así igualmente, agrandar, reducir, sustituir, ejemplos de estos los cambios en envases de diversos productos que hoy se ofertan en el mercado y antes tenían otra constitución. (Schoening, 2017).

Schnarch, (2014) comenta que la innovación implica dos instancias: una creativa, de búsqueda y generación de ideas, y la otra ejecutiva, que transforma y modifica. De esta forma, todo proceso innovador comienza por el surgimiento de esa idea, y es, por tanto, un proceso creativo que termina con la puesta en marcha de la misma. "...la creatividad se mide por el resultado final, una idea, un nuevo producto, una nueva manera de hacer algo y aún cuando todos los seres humanos pueden ser creativos, la creatividad es una característica de personas que se autorealizan". (p. 26). Que existan personas más creativas que otras depende, entre otros aspectos, de las características propias de su personalidad, del medio en donde se hayan desarrollado, de factores sociales y culturales, e incluso motivacionales.

Si bien, los seres humanos tienen la capacidad de ser creativos en mayor o menor 


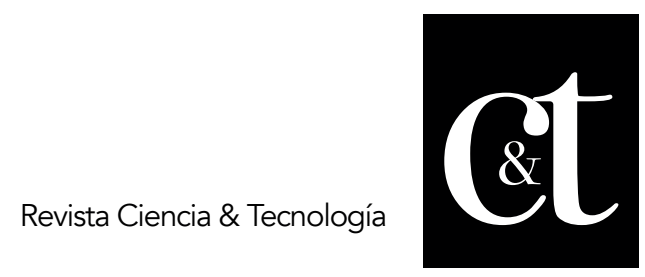

No. 26, 30 de abril de 2020

ISSN impreso: 1390 - 6321

ISSN online: 2661 - 6734

medida, la creatividad «no es algo que exista en abstracto, sino que es un juicio sociocultural acerca de la novedad, la adecuación, la calidad y la importancia de un producto». Por lo tanto, su trascendencia e importancia depende de la valoración o el impacto que tenga sobre terceros, por lo que una idea puede perderse simplemente por no haber sido acogida. A diferencia de muchos atributos mentales, no está relacionada con normas preestablecidas ni con la lógica. (Rajadell, 2019).

\section{Emprendimiento y el Valor Añadido por Innovación}

Al igual que los procesos innovadores, un emprendimiento empresarial surge de una idea preconcebida para desarrollar un nuevo negocio, con lo cual propicia satisfacer una carencia o demanda detectada en el mercado. Rajadell (2019) afirma que al igual que toda actividad humana comienza con una idea, la creación de una empresa no es ninguna excepción. No es imprescindible ser el gran inventor para crear un nuevo emprendimiento, pues en la gran mayoría de los casos no se parte de grandes descubrimientos técnicos, sino de la simple observación del entorno y de las oportunidades que éste puede brindar, sólo siendo capaces de visualizarlas.

Se dice que el emprendimiento es aquel proceso que permite a una persona o grupo de ellas, alcanzar nuevos retos mediante el desarrollo de un nuevo proyecto, negocio o ideas que generen aumento de valor para el mercado, y es, además, fuente generadora no sólo de ingresos, sino además de beneficios a la sociedad mediante la creación de empleo. Un proceso de emprendimiento contribuye a brindar oportunidades de crecimiento profesional a personas que, por diversos motivos, encuentran en el mismo el cumplimiento de un sueño, pues pueden desarrollar sus habilidades y capacidades aportándoles a sus vidas un sentido diferente.

El emprendimiento tiene su origen en los inicios de la humanidad, ya que el hombre siempre se ha caracterizado por asumir riesgos para proveerse a sí mismo y a su familia de mejores condiciones de vida. En la actualidad, con las constantes crisis económicas y el consecuente efecto negativo que genera en todos los ámbitos de la vida humana, surgen emprendedores por necesidad más que por vocación y es aquí donde, en ocasiones, estos emprendimientos parten de las ideas más innovadoras y efectivas.

El término emprendimiento fue definido por primera vez por el economista anglo francés Richard Cantillon (1755) quien describió al emprendedor como "la persona que paga un cierto precio para revender un producto a un precio incierto, por ende, tomando decisiones acerca de la obtención y uso de recursos, admitiendo consecuentemente el riesgo. (Moreno y Tercila, 2016) Cantillon define al entrepreneur como el "agente que compra los medios de producción a ciertos precios y los combina en forma ordenada para obtener de allí un nuevo producto". (Schnarch, 2014, p. 21).

Rajadell (2019) comenta que el emprendedor es el responsable de crear una empresa a partir de sus capacidades, sus habilidades y sus recursos para detectar oportunidades de negocio. Los recursos no necesariamente han de ser económicos, ya que hay célebres casos de emprendedores que partieron de 


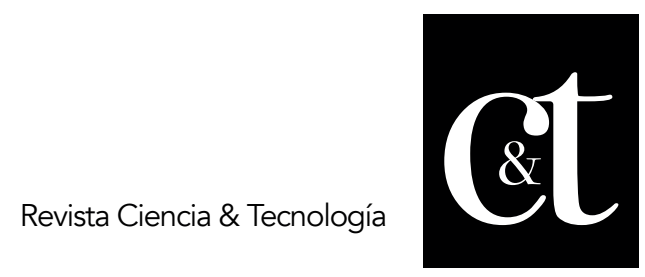

No. 26, 30 de abril de 2020

ISSN impreso: 1390 - 6321

ISSN online: 2661 - 6734

situaciones desfavorecidas, como puede ser el garaje de sus casas: Amazon, Apple, eBay, Google, Harley Davidson, Hewlett Packard, Mattel, Microsoft, Nike, YouTube, son ejemplos de emprendimientos que surgieron casi desde la nada.

El emprendedor tiene la semilla del empresario, por eso muchas veces son la misma persona; ambos persiguen objetivos similares: la obtención de beneficios, la generación de empleo, sin embargo, el emprendedor puede o no llegar a ser el empresario, y ello radica en que el primero quiere llevar a cabo una idea y poner en práctica ese sueño, sin embargo le pueden faltar las herramientas profesionales y de experiencia para hacer lo que el empresario hace, gestionar el proyecto una vez implementado.

De esta manera, es importante mencionar que el emprendedor empresarial necesita de los conocimientos de las ramas administrativas y afines, ya que por muy buena idea o proyecto que tenga, si, al ponerlo en práctica, no conoce a su público objetivo, sus hábitos de consumo, su comportamiento de compra, los procesos de logística y distribución, los de comunicación, así como todas las cuestiones propias de la puesta en marcha de dicho negocio, fracasará, ya que caen en una visión miope de la realidad del mercado, sin tener en cuenta las leyes del Marketing y cómo funciona el mundo empresarial competitivo. Aunque muchos emprendedores comienzan de manera empírica, se ha comprobado que el tener un nivel de preparación y de estudios o tener una determinada experiencia profesional, posibilita mayores oportunidades de prosperar en el intento. (Rajadell, 2019).

En el proceso de creación de una empresa, o de emprender en si mismo, encontrar ideas es el inicio. Al igual que los innovadores, los emprendedores tienden a pensar no sólo creativamente, sino, además, ellos logran llevar a cabo las ideas que luego se convierten en proyectos empresariales mediante verdaderos procesos creativos e innovadores. El emprendedor es capaz de detectar cuáles serán estas ideas que, llevadas a la práctica tendrán la acogida esperada, generando los beneficios posteriores correspondientes y ello sólo se logra mediante un proceso verdaderamente creativo.

No sólo la creación de un proyecto empresarial requiere la puesta en práctica de esa idea creativa, sino que, a su vez, la continuidad del mismo está ligado de manera indisoluble a los procesos de innovación, que conllevan a su vez una alta y constante creatividad en su funcionamiento. Si los emprendedores empresariales quieren asegurar su permanencia en el mercado, y el florecimiento de su negocio, deben propiciar el desarrollo de la creatividad y la innovación desde el corazón de su organización. La innovación puede llegar a ser una verdadera fuente de ventajas competitivas, ya que permite, mediante la detención de oportunidades, la creación de ofertas diferentes y valiosas para la sociedad.

En muy pocas ocasiones se puede iniciar un negocio o proyecto empresarial sin innovar, sólo en casos en que exista una excesiva demanda de un producto o servicio específico se puede comenzar y mantener un emprendimiento, ofertando lo ya existente, o sea, más de lo mismo. Sin embargo, en la actualidad son pocos los emprendedores que pueden sentir que no tienen amenazas competitivas importantes, ya que la hostilidad del entorno económico y empresarial actual es 


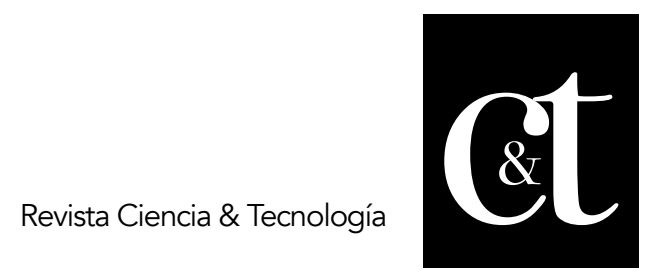

No. 26, 30 de abril de 2020

ISSN impreso: 1390 - 6321

ISSN online: 2661 - 6734

tal, que no queda de otra que actuar en función de las dinámicas del mercado.

A la larga, este hacer igual que otros, sólo trae como consecuencia que la oferta y la demanda se equilibren, y en el peor de los casos, la demanda comienza a disminuir, por lo que sólo persisten entonces, aquellas organizaciones con verdaderas ventajas competitivas. Es importante, por tanto, no confiarse en este camino del emprendimiento empresarial, aunque un mercado específico no haya sido satisfecho, por ello la innovación asegura un futuro venturoso para aquellos negocios que quieren llegar lejos.

Schnarch (2014), comenta que "si este mismo emprendedor entra en un mercado desatendido ofreciendo esos mismos productos o servicios con mejoras añadidas - cambios significativos en cualquiera de sus fases de producción o comercialización, con el propósito de dar resultados de mayor valor, ya está innovando. O sea, ofertar bienes o servicios mediante nuevas estrategias de Marketing, o simplemente atender un nuevo mercado, implica ya un proceso de innovación" (p. 32).

También a lo interno de una organización, se le reconocen formas de emprendimiento de sus trabajadores mediante la creación de nuevos bienes o servicios, nuevos procesos y mejoras a la producción, o a la utilización de materias primas, materiales e insumos de producción que traen como consecuencia ofertas de mejor valor y en muchos casos la creación de nuevas ventajas competitivas.

De esta manera, la creatividad e innovación requieren de personas emprendedoras con coraje y corazón, tanto al interior de todo tipo de organizaciones, públicas o privadas, capaces de cambiar y mejorar productos, procesos, métodos o sistemas para hacer crecer las empresas, así como personas con espíritu empresarial que creen sus propias empresas, para plasmar sus visiones y generar empleo y progreso. (Schnarch, A. 2014, p. 37).

\section{Conclusión}

La innovación conlleva dos fases: la creativa, que es la búsqueda de ideas y la ejecutiva, que es cuando se transforman las mismas en una oferta que satisface una necesidad, deseo o demanda. El innovador es quien ve el lado práctico de la idea generada y por tanto no le importa el riesgo asociado, es capaz de llevar a cabo esas ideas asumiendo con total valentía y responsabilidad las consecuencias, igualmente el emprendedor es quien, con su ímpetu, habilidades y coraje, pone en práctica esta innovación y se arriesga en el proceso de hacerle ver su público objetivo, que la oferta de valor va a resolver su problema satisfaciendo esa necesidad o deseo específico. Si este emprendedor, además, cuenta con las capacidades y habilidades empresariales necesarias para llevar a cabo su proyecto, tendrá altas probabilidades de éxito en su empeño. Ambos procesos, innovación y emprendimiento están estrechamente relacionados, siendo llevados a cabo, muchas veces, por la misma persona. Hoy, por tanto, es un imperativo de las sociedades y los gobiernos, desarrollar en sus ciudadanos, estas capacidades creativas y potenciar el emprendimiento innovador.

La innovación, es el denominador común al perfil emprendedor. Al mejorar lo 


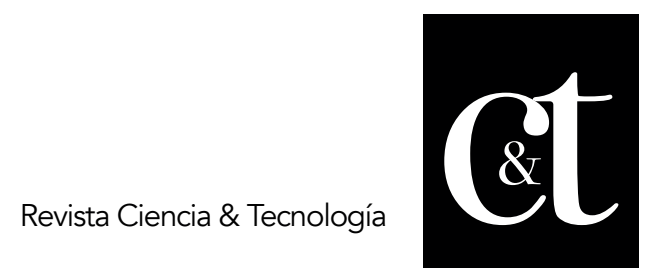

No. 26, 30 de abril de 2020

ISSN impreso: 1390 - 6321

ISSN online: 2661 - 6734

existente, al aumentar el valor de lo que se oferta, al crear soluciones que satisfacen deseos y demandas insatisfechas o resuelven diversos problemas, se innova, y se corren riesgos asociados a este proceso. El emprendedor utiliza la innovación para solucionar precisamente estos problemas e incluso, identificar oportunidades de mercados que conllevan a la creación de nuevas empresas o negocios, posibilitando la creación de nuevos empleos y el logro de un sueño. Sólo estas personas emprendedoras e innovadoras a su vez, logran asumir con valentía estos riesgos y aunque lleguen o no a triunfar en el proceso, ya llevan un gran reconocimiento y apoyo por ser parte del avance económico de un país, y consecuentemente del bienestar social y general de su población.

\section{Referencias bibliográficas}

Fuentelsaz L. y Montero J. (2015). ¿Qué hace que algunos emprendedores sean más innovadores? Universia Business Review, núm. 47, julio-septiembre, pp. 14-31.

Moreno, C. y Tercila, F. (2016). Emprendimiento y plan de negocio, RIL editores, 2016. ProQuest Ebook Central. Recuperado de: [http://ebookcentral.proquest.com/lib/bibliosudamericanocuencasp/detail.ac tion? docID $=4675975$. ]

Perazzo, D. (1996). Implicaciones de la Innovación. En: Actas del V Congreso del Mercosur, 1996. Buenos Aires.

Pizzi A. y Brunet. I (2013). Creación de empresas, modelos de innovación y pymes. Cuadernos del Cendes. vol.30 no.83 Caracas ago. pp. 53-74.

Rajadell, M. (2019). Creatividad: emprendimiento y mejora continua, Editorial Reverté. Recuperado de: [http://ebookcentral.proquest.com/lib/bibliosudamericanocuencasp/detail.ac tion? docID $=5809940$.]

Schnarch, A. (2014). Emprendimiento exitoso: cómo mejorar su proceso y gestión. Bogotá. Colombia. ECOE Ediciones.

Schoening, A: (2017). iQuiero mi propio negocio! Una guía para lograrlo con lo que sabe y con lo que tiene. México. Editorial Trillas. 